\title{
Serum Procalcitonin and Proinflammatory Markers in Polycystic Ovary Syndrome
}

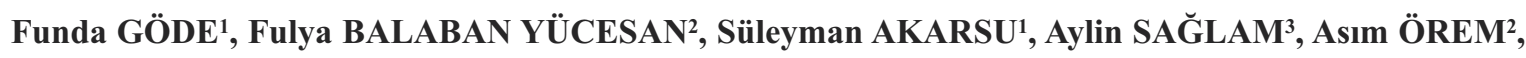 \\ Khayal SHARAFKHANOV ${ }^{3}$ \\ Izmir, Turkey
}

\begin{abstract}
OBJECTIVE: We evaluated levels of procalcitonin and proinflammatory markers in patients with polycystic ovary syndrome and compared them with controls in the Black Sea region of Turkey.

STUDY DESIGN: This prospective controlled study involved patients with polycystic ovary syndrome $(n=59)$ and healthy age-matched controls $(n=26$; total, $n=85)$. Serum procalcitonin, white blood cells , high-sensitivity $\mathrm{C}$-reactive protein, homocysteine levels, insulin resistance, and lipid profiles were compared between the polycystic ovary syndrome and control groups. The same parameters were also compared between overweight and normal-weight polycystic ovary syndrome patients.

RESULTS: Serum procalcitonin, homocysteine, high-sensitivity C-reactive protein, and white blood cell levels were similar in the polycystic ovary syndrome and control groups. High-density lipoprotein levels were lower in the polycystic ovary syndrome group than in the control group $(p<0.05)$. In a subgroup analysis of the polycystic ovary syndrome group, there were no significant differences between overweight and normal-weight polycystic ovary syndrome patients with regard to proinflammatory markers (serum white blood cell, high-sensitivity C-reactive protein, homocysteine, procalcitonin levels). However, total cholesterol, low-density lipoprotein, and triglyceride levels were significantly higher in overweight polycystic ovary syndrome patients $(p<0.005)$. Serum high-density lipoprotein levels were significantly lower in the overweight polycystic ovary syndrome group than in the normal-weight group $(p<0.005)$. Fasting insulin and HOMA-IR levels were significantly higher in overweight polycystic ovary syndrome than normal-weight polycystic ovary syndrome patients $(p<0.05)$.
\end{abstract}

CONCLUSIONS: Serum procalcitonin, high-sensitivity C-reactive protein, white blood cell, and homocysteine levels were within normal ranges in polycystic ovary syndrome patients. These results may be related to the relatively young age and regional differences in the study group.

Keywords: Procalcitonin, Homocysteine, Polycystic ovary syndrome, High-sensitivity C-reactive protein

Gynecol Obstet Reprod Med 2017;23(1):20-25

${ }^{1}$ Izmir University School of Medicine Department of Obstetrics and Gynecology, İzmir

${ }^{2}$ Karadeniz Technical University Scholl of Medicine Department of Biyochemistry, Trabzon

${ }^{3}$ Tekden Hospital Department of Obstetrics and Gynecology , Istanbul

Address of Correspondence: Funda Göde

Izmir University School of Medicine

Department of Obstetrics and

Gynecology İzmir, Turkey

funda.gode@gmail.com

Submitted for Publication: $\quad$ 20.07.2016

Accepted for Publication: $\quad 21.12 .2016$

\begin{tabular}{|c|c|}
\hline & Access this article online \\
\hline $\begin{array}{c}\text { Quick Response Code: } \\
\text { 口as }\end{array}$ & Website: www.gorm.com.tr \\
\cline { 2 - 2 } & DOI:10.21613/GORM.2016.619 \\
\hline
\end{tabular}

How to cite this article: Göde F. Balaban Yücesan F. Akarsu S. Sağlam A. Örem A. Sharafkhanov K. Serum Procalcitonin and Proinflammatory Markers in Polycystic Ovary Syndrome. Gynecol Obstet Reprod Med 2017; 23(1):20-25

\section{Introduction}

Polycystic ovary syndrome (PCOS) is, in fact, a heterogeneous collection of symptoms and signs. They form a disorder with a spectrum that ranges from causing severe reproductive disturbances in some women to affecting endocrine and metabolic function in others. It is the most common endocrine disorder, affecting $5-10 \%$ of younger women (1). While there are many extra-ovarian aspects to the pathophysiology of PCOS, ovarian dysfunction is central.

At a joint European Society of Human Reproduction and Embryology/American Society for Reproductive Medicine (ESHRE/ASRM) consensus meeting, a refined definition for PCOS was agreed upon. Two of the following three criteria are required to make the diagnosis: oligomenorrhea and/or oligo/anovulation, hyperandrogenism (clinical and/or biochemical, with exclusion of other etiologies such as congenital adrenal hyperplasia, androgen-secreting tumors, Cushing syndrome), and polycystic ovaries (The Rotterdam 
ESHRE/ASRM-sponsored PCOS consensus workshop group, 2004) (2). There have been many studies demonstrating the presence of insulin resistance and corresponding hyperinsulinemia in both obese and non-obese women with PCOS (3).

Obesity and metabolic abnormalities are recognized risk factors for the development of cardiovascular disease in the general population, and these are also recognized features of PCOS (3). The basis for the idea that women with PCOS are at greater risk of cardiovascular disease is that these women are more insulin resistant than weight-matched controls, and that metabolic disturbances associated with insulin resistance increase cardiovascular risk in other population groups. Moreover, in patients with PCOS, circulating levels of many proinflammatory markers related to cardiovascular disease, such as TNF $\alpha$, IL 6, h-CRP, homocysteine, white blood cell (WBC) counts, and neutrophil counts, are increased compared to age- and/or body mass index (BMI)-matched controls (4-7).

Procalcitonin (PCT) is a 116-amino-acid polypeptide that is the precursor of calcitonin. It is produced not only by neuroendocrine cells of the thyroid gland and K-cells of the lung, but also by several other cell types and in many organs in response to proinflammatory stimuli (8). Serum PCT levels are very low or undetectable in the healthy state because most PCT is processed into calcitonin intracellularly in the Golgi apparatus (9). Recently, elevated plasma PCT levels in the normal range have been associated with measures of obesity, insulin resistance, and other metabolic syndromes (10). It has also been shown that adipose tissue is capable of both expressing and secreting PCT $(11,12)$. These findings suggest that procalcitonin might be a useful marker for obesity-related low-grade inflammation and PCOS.

Although many studies support an increased cardiovascular risk in women with PCOS, some have found no correlation and the evidence is still limited. Indeed, it remains unclear whether all women with PCOS are at risk for cardiovascular diseases. Thus, in this study, we examined the levels of serum PCT and other proinflammatory markers in women with PCOS in the Black Sea region of Turkey.

\section{Material and Method}

This was a prospective controlled study performed between January 2012 and 2013 in the Obstetrics and Gynecology department of Rize Government Hospital, Turkey. The study involved women with PCOS $(\mathrm{n}=59)$ and healthy women $(\mathrm{n}=$ 26; total, $n=85$ ). The diagnosis of PCOS was made using the Rotterdam criteria, 2004 (2). At least two of the following three criteria needed to be present: oligomenorrhea and/or oligo/anovulation, hyperandrogenism (clinical and/or biochemical), and polycystic ovaries. The control group consisted of women with regular menstrual cycles, normal serum biochemical parameters, and no clinical sign of hyperandrogenism (biochemical or clinical). The control and PCOS groups were matched for age and BMI. The study was approved by the Ethical Committee of Recep Tayyip Erdogan University. Informed consent was obtained from all of the women who participated. We assessed clinical and anthropometric variables, including blood pressure and BMI in all of the participants. BMI was defined as the body mass divided by the square of the body height and is expressed in $\mathrm{kg} / \mathrm{m}^{2}$. The PCOS patients were sub grouped according to BMI as overweight (BMI $\geq 25 \mathrm{~kg} / \mathrm{m}^{2}$ ) or normal weight $\left(\mathrm{BMI}<25 \mathrm{~kg} / \mathrm{m}^{2}\right)$ patients. The Ferriman-Galwey score was used to evaluate hirsutism. A score $\geq 8$ is considered indicative of excess androgen (13).

\section{Exclusion criteria}

Patients with congenital adrenal hyperplasia, Cushing's syndrome, androgen-secreting tumors, hyperprolactinemia, and liver, kidney, and thyroid diseases were excluded by biochemical testing. In addition, patients with a known history of hypertension, hyperlipidemia, or diabetes were not included. Smokers, regular intense exercise performers, and patients who had received hormone therapy in the previous 6 months were also excluded.

\section{Biochemical measurements}

Fasting serum insulin, glucose, FSH, LH, estradiol, total testosterone, free testosterone, sex hormone binding globulin (SHBG), 17-hydroxyprogesterone, dehydroepioandrostenedione sulfate (DHEAS), total cholesterol, h-CRP, Hcy, triglyceride, HDL, LDL, procalcitonin, and WBCs were measured in all of the women. Blood samples were drawn from all of the subjects during the early follicular phase of the menstrual cycle after an overnight fast. Fasting blood glucose was measured using the glucose oxidase method (Cobas Integra 400 Plus, Roche Diagnostics, Germany). We measured fasting serum insulin, total testosterone, estradiol, FSH, LH, and DHEAS concentrations using an electro-chemiluminescence method (Elecys 2010 Hitachi, Roche Diagnostics). Serumfree testosterone and 17-hydroxyprogesterone levels were measured using a high sensitivity enzyme-linked immunosorbent assay (ELISA).

Venous blood samples were centrifuged (4000 g, $10 \mathrm{~s})$ and plasma was stored at $80^{\circ} \mathrm{C}$ until PCT analysis. PCT was measured with an ultrasensitive immunoassay using time-resolved amplified cryptate emission (TRACE) technology (Kryptor; Brahms). This assay measures concentrations between 0.02 and $5000 \mathrm{ng} / \mathrm{mL}(0.02-50 \mathrm{ng} / \mathrm{mL}$ directly and up to 5000 $\mathrm{ng} / \mathrm{mL}$ after sample dilution). The detection limit calculated using the imprecision profile was assessed at $0.019 \mathrm{ng} / \mathrm{mL}$ with a $95 \%$ probability. The functional assay sensitivity, defined as the lowest analyte concentration that could be determined with an inter assay coefficient of variation $<20$, was assessed at $0.06 \mathrm{ng} / \mathrm{mL}$ with a $95 \%$ probability. Homocysteine levels were measured using the chemiluminescence method (Immulite 1000 analyzer, Siemens, ABD). Total cholesterol, HDL, LDL, triglyceride, glucose, and h-CRP levels were determined with immunoassay kits (Abbott Architect c16000). 
LDL cholesterol level was calculated using the Friedwald formula (LDL cholesterol $=$ total cholesterol - [VLD + HDL] $)$ (14). The insulin resistance was estimated to be the HOMA score calculated with the formula: (fasting serum insulin $(\mathrm{IU} / \mathrm{mL}) \times$ fasting plasma glucose $(\mathrm{mmol} / \mathrm{L}) / 22.5)$.

\section{Statistical analysis}

The SPSS software (ver. 17.0; SPSS Inc., Chicago, IL) was used for statistical analyses. Data are presented as means \pm SDs and raw numbers, unless otherwise stated. Comparisons between groups were performed with the independent sample t-test for parametric conditions. Correlation analysis was used and the Spearman correlation coefficient was calculated to detect any correlation between proinflammatory parameters and other variables. A p value $<0.05$ was considered statistically significant.

\section{Results}

In total, 85 patients were included in the study (PCOS group, $\mathrm{n}=59$, and control group, $\mathrm{n}=25$ ).

\section{Polycystic ovary syndrome and control groups}

Anthropometric, metabolic, and hormonal features of women with PCOS and controls are shown in table 1. There were no differences between the groups in age or BMI (age: $24.25 \pm 6.4$ years and mean BMI:26.55 \pm 6.83 vs. age: $26.88 \pm 6.50$ years and mean BMI: $25.78 \pm 4.43$ respectively). The Ferriman-Gallwey scores of PCOS patients were significantly higher than the control group $(\mathrm{p}<0.05)$. Regarding biochemical parameters, $\mathrm{LH}$, free testosterone, total testosterone levels, and $17-\mathrm{OH}$ progesterone were significantly higher in the PCOS group $(\mathrm{p}<0.05)$ and HDL levels were significantly lower $(\mathrm{p}<0.05)$. The other biochemical parameters, including FSH, E2, SHBG, DHEAS, LDL, total cholesterol, triglyceride, and glucose, did not differ between the groups. Regarding proinflammatory markers, serum h CRP, homocysteine, and PCT levels did not differ between PCOS patients and control subjects. When the correlation analysis was evaluated, we detected a significant correlation between PCT levels and HOMA-IR, BMI, and insulin levels $(p<0.05)$. There was a significant correlation between WBC levels and insulin, BMI, HOMA-IR, and triglyceride levels $(p<0.05)$. There was a significant negative correlation between HDL levels and BMI, insulin, HOMA-IR, triglycerides, and total cholesterol levels ( $\mathrm{p}<0.05$ ). There was no correlation between homocysteine levels, CRP levels, and other variables.

Table 1: Anthrophometric, metabolic and hormonal features of women with polycystic ovary syndrome and controls

\begin{tabular}{|c|c|c|c|}
\hline & $\begin{array}{l}\text { Healthy control } \\
(n: 26)\end{array}$ & $\begin{array}{l}\text { Polycystic ovary syndrome } \\
\text { (n:59) }\end{array}$ & $p$ \\
\hline Age (yr) & $26.88 \pm 6.50$ & $24.25 \pm 6.40$ & 0.086 \\
\hline BMI $\left(\mathrm{kg} / \mathrm{m}^{2}\right)$ & $25.78 \pm 4.43$ & $26.55 \pm 6.83$ & 0.620 \\
\hline Waist-hip ratio & $0.76 \pm 0.08$ & $0.76 \pm 0.06$ & 0.842 \\
\hline Ferriman-Gallwey score & $3.67 \pm 2.51$ & $9.23 \pm 3.37$ & 0.000 \\
\hline $\mathrm{FSH}(\mathrm{mlU} / \mathrm{mL})$ & $6.00 \pm 1.21$ & $5.41 \pm 1.46$ & 0.082 \\
\hline LH (mIU/mL) & $6.38 \pm 4.06$ & $10.03 \pm 6.34$ & 0.011 \\
\hline Total testosterone $(\mathrm{ng} / \mathrm{mL})$ & $0.28 \pm 0.13$ & $0.41 \pm 0.14$ & 0.001 \\
\hline Free testosterone $(\mathrm{pg} / \mathrm{mL})$ & $1.38 \pm 0.69$ & $2.01 \pm 0.89$ & 0.003 \\
\hline SHBG (nmol/L) & $51.26 \pm 31.73$ & $42.77 \pm 24.68$ & 0.266 \\
\hline DHEA-S (ug/mL) & $240.75 \pm 106.90$ & $283.54 \pm 127.88$ & 0.162 \\
\hline 17-OHP (ng/mL) & $0.73 \pm 0.48$ & $0.98 \pm 0.45$ & 0.037 \\
\hline Free androgen index $(U)$ & $0.76 \pm 0.62$ & $1.31 \pm 1.14$ & 0.066 \\
\hline Total cholesterol (mg/dL) & $175.75 \pm 32.64$ & $169.61 \pm 32.35$ & 0.442 \\
\hline LDL cholesterol (mg/dL) & $116.58 \pm 33.96$ & $114.82 \pm 33.80$ & 0.833 \\
\hline HDL cholesterol (mg/dL) & $59.14 \pm 12.12$ & $52.55 \pm 11.05$ & 0.021 \\
\hline Triglycerides (mg/dL) & $93.29 \pm 55.65$ & $87.90 \pm 55.76$ & 0.694 \\
\hline Fasting blood glucose (mg/dL) & $92.08 \pm 9.47$ & $92.78 \pm 9.31$ & 0.759 \\
\hline Fasting blood insulin(uU/mL) & $10.92 \pm 13.62$ & $10.26 \pm 7.52$ & 0.789 \\
\hline Glucose/Insulin ratio & $12.89 \pm 6.56$ & $12.67 \pm 6.00$ & 0.891 \\
\hline HOMA-IR & $2.59 \pm 3.44$ & $2.44 \pm 2.00$ & 0.820 \\
\hline WBC (cellx103/uL) & $5.62 \pm 1.19$ & $6.26 \pm 1.57$ & 0.082 \\
\hline hs-CRP (ug/mL) & $3.52 \pm 1.41$ & $3.65 \pm 1.96$ & 0.769 \\
\hline Homocystein (umol/L) & $12.12 \pm 6.65$ & $14.20 \pm 8.59$ & 0.291 \\
\hline PCT (ng/mL) & $0.028 \pm 0.02$ & $0.026 \pm 0.01$ & 0.703 \\
\hline
\end{tabular}

BMI: Body mass index, SHBG: Sex hormone binding globüline, LDL: Low density lipid, HDL: High density lipid, IR: Insulin resistance, WBC: White blood cell, hs: High sensitive, PCT: Procalcitonin 


\section{Overweight and normal-weight group in polycystic ovary} syndrome patients

The PCOS group patients were split according to BMI and grouped as overweight (BMI $\geq 25 \mathrm{~kg} / \mathrm{m}^{2}$ ) and normal-weight $\left(\right.$ BMI $\left.<25 \mathrm{~kg} / \mathrm{m}^{2}\right)$ patients. When a subgroup analysis was performed in the PCOS group, there were no significant differences between overweight and normal-weight PCOS patients in terms of proinflammatory markers (serum WBC, h CRP, homocysteine, PCT levels). However, total cholesterol, LDL, and triglycerides were significantly higher in overweight PCOS patients (Figure 1). Fasting insulin and HOMA-IR were also significantly higher in overweight PCOS patients $(\mathrm{p}<$ 0.05; Figure 2).

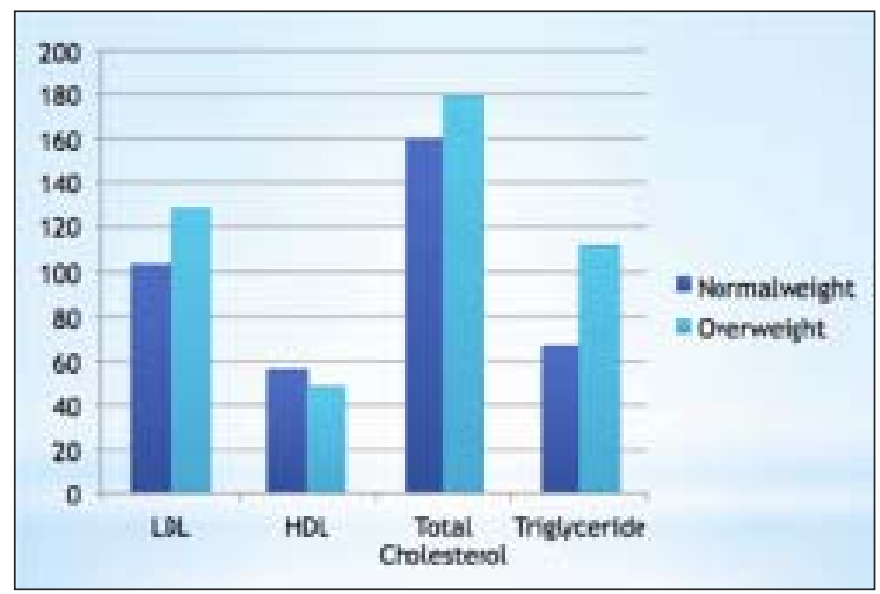

Figure 1: Lipid profile of overweight and normal weight polycystic ovary syndrome patients

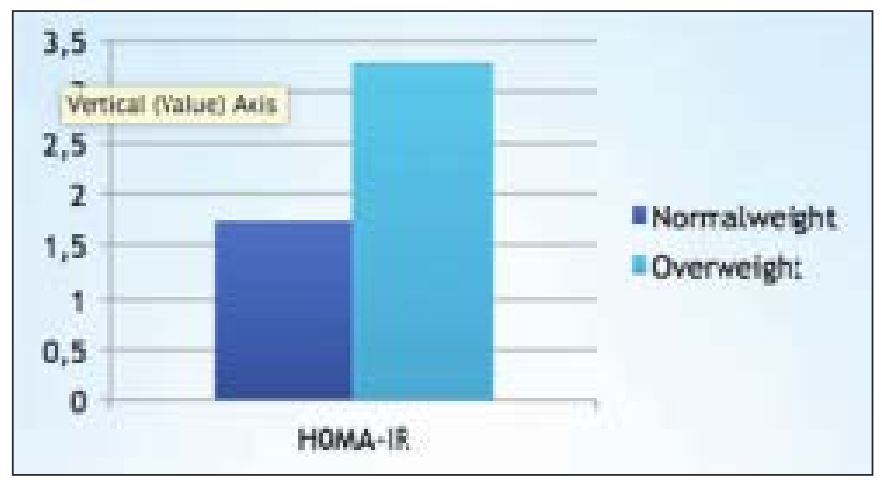

Figure 2: Fasting insulin and HOMA-IR levels of overweight and normal weight polycystic ovary syndrome patients

\section{Discussion}

Although PCOS has been associated with cardiovascular risk factors, including obesity, hypertension, dyslipidemia, and impaired glucose tolerance, the exact cardiovascular risk has not been clearly identified (15). Because of the wide range of symptoms in this disease, it has patients with different clinical findings. Thus, not all of the metabolic features may accompany the disease process and some subgroups may not be at risk for cardiovascular disease. Cardiovascular risk bio- markers may be helpful in clinically diagnosing those PCOS patients who have cardiovascular risk or disease.

Elevated plasma Hcy is considered a risk factor for cardiovascular disease. Many studies have focused on the association between plasma Hcy levels and PCOS, and some have reported higher levels of Hcy in PCOS patients than controls (16-18). In PCOS patients, there is a correlation between plasma levels of Hcy and androgen (19). Maleedhu et al. reported increased Hcy levels in obese women with PCOS (20). In contrast, Kilic-Okman et al. found that insulin resistance, dyslipidemia, and BMI did not correlate with serum Hcy levels in PCOS patients (21). In our study, we found no significant increase in Hcy levels in PCOS patients compared to the control group. We also found no significant difference in Hcy levels between overweight and normal-weight patients. Some connections have been made between hyperhomocysteinemia and elevated risk for cardiovascular events; however, the role of plasma Hcy in cardiovascular disease remains unclear. However, it is known that plasma Hcy increases with aging and is associated with other health-related conditions (22); thus, our findings may be related to the relatively young age of our study population.

In terms of the lipid profile, our findings in PCOS patients were similar to those of Fulghesu et al., who reported no increase in the profile (23). In our study, both overweight women and PCOS patients had significantly lower levels of HDL cholesterol. We also found that BMI was strongly correlated with a 'worse' lipid profile. Our results demonstrated significant elevations in parameters reflecting hyperandrogenism (higher serum levels of total testosterone, free testosterone) in PCOS patients, whereas SHBG levels were lower in women with PCOS than in the controls. We also found significant increases in serum levels of glucose, insulin, and HOMA-IR scores in overweight PCOS patients than normalweight PCOS patients showing the importance of BMI in PCOS patients.

Low-grade chronic inflammation, reflected by elevated serum levels of CRP, has been closely linked to insulin resistance, central obesity, and a higher risk of cardiovascular complications in PCOS patients. Many studies have focused on proinflammatory markers including serum PCT, CRP, WBC, and neutrophil counts, in PCOS patients. Although Sumitran et al. found that CRP levels were higher in PCOS patients than controls, Pedroso et al. reported no significant increases in CRP levels in PCOS patients $(24,25)$. We also detected no difference in CRP levels between PCOS patients and controls.

Procalcitonin is widely used as a biomarker in bacterial infection and sepsis. It may be useful with regard to the inflammatory theory of PCOS (26). Generally, in humans and animal models, PCT levels correlate with the severity of a condition and remain elevated for the duration of the inflammatory process (27). Indeed, high PCT levels tend to last as long as 
the inflammatory process and appear to correlate with the outcome of the illness (28). There are reports of elevated PCT in the presence of sepsis, respiratory tract infections, and other 'traditional' infectious diseases. Elevated PCT levels have been reported in patients with cirrhosis, pancreatitis, mesenteric infarction, cardiogenic shock, and hypotension during surgery (29). Puder et al. found elevated CRP, WBC, and PCT levels in women with PCOS; however, we did not find increased levels in PCOS patients compared to the controls (26). Thus, our results in PCOS patients differ from those reported by Puder et al., Rashad et al. and Repaci et al (26,30,31). The mean age of PCOS patients in our study was younger than that of patients in the studies by Puder et al. and Repaci et al $(26,30)$. With advancing age, a slight increase in CRP and cytokines was detected (32). The increase in serum levels of proinflammatory cytokines and CRP could also be the result of the decline in kidney function that is observed with increasing age (33). It is also proposed that higher inflammatory marker levels are related to an increased volume of adipose tissue, declining sex hormones, and increasing oxidative stress (34).

It remains unclear why some PCOS patients have cardiovascular risk factors and others do not. The heterogeneous population with this disease may be a factor. Thus, obesity, metabolic syndrome, and insulin resistance are not criteria used to define the disease. Some study population subgroups, including patients with central obesity and insulin resistance, may have cardiovascular disease risk factors; other subgroups, including normal weight and normoinsulinemic patients, may not. This merits further investigation. Moreover, disease procession may affect metabolic features over the long term of this chronic condition, which may be important in older women. Environmental conditions and lifestyle are other important factors in cardiovascular disease process. Thus, population differences should also be taken into account, due to regional differences in cytokine levels, genotypes, and polymorphism (35). In addition, diet is believed to have a major influence on inflammatory signaling. This could regulate metabolic pathways and bioenergetics in a way that could be translated into a stable epigenetic pattern of gene expression (36). Our study population was from Black Sea region of Turkey, which may be a factor in terms of dietary habits and metabolic features of the disease.

Although we found no significant difference in PCT levels between the PCOS and control groups, there was a positive correlation among PCT levels, BMI, and HOMA-IR and a negative correlation with HDL levels. These findings suggest a relationship between features of metabolic syndrome and proinflammatory markers, which should be clarified. In our study, the PCOS and control groups were equally obese. This may a reason for the similar results. However, when we performed a subgroup analysis in the PCOS group according to BMI, we also found no significant difference in proinflammatory markers between overweight and normal-weight PCOS patients. A limitation of our study is the relatively small sample size. Subgroup analysis including larger populations may be helpful in better evaluating cardiovascular risk factors in PCOS and drawing more definitive conclusions on the matter.

In conclusion, we detected no difference in CRP, WBC, PCT, or Hcy levels between PCOS patients and controls. Many factors (e.g., diet, renal function, age, population differences, severity, and duration of inflammation) may influence serum levels of these proinflammatory markers. Because the clinical features of PCOS patients are very heterogeneous, larger, longer-term studies are needed to determine the reasons for reported differences and to draw definitive conclusions on the subject.

\section{References}

1. Asuncion M, Calvo RM, San Millan JL, Sancho J, Avila $\mathrm{S}$, Escobar-Morreale HF. A prospective study of the prevalence of the polycystic ovary syndrome in unselected Caucasian women from Spain. J Clin Endocrinol Metab 2000;85(7):2434-8

2. Rotterdam ESHRE/ASRM-Sponsored PCOS Consensus Workshop Group. Revised 2003 consensus on diagnostic criteria and long-term health risks related to polycystic ovary syndrome. Fertil Steril 2004;81(1):19-25.

3. Balen AH, Rajkowha M. Polycystic ovary syndrome: a systemic disorder? Best Pract Res Clin Obstet Gynecol 2003;17(2):263-74.

4. Kelly CC, Lyall H, Petrie JR, Gould GW, Connell JM, Sattar N. Low grade chronic inflammation in women with polycystic ovarian syndrome. J Clin Endocrinol Metab 2001;86(6):2453-5.

5. Ibanez L, Jaramillo AM, Ferrer A, de Zegher F. High neutrophil count in girls and women with hyperinsulinaemic hyperandrogenism: Normalization with metformin and flutamide overcomes the aggravation by oral contraception. Hum Reprod 2005;20(9):2457-62.

6. Dahlgren E, Janson PO, Johansson S, Lapidus L, Oden A. Polycystic ovary syndrome and risk for myocardial infarction. Evaluated from a risk factor model based on a prospective population study of women. Acta Obstet Gynecol Scand 1992:71(8):599-604.

7. Talbott E, Guzick D, Clerici A, Berga S, Detre K, Weimer $\mathrm{K}$ et al. Coronary heart disease risk factors in women polycystic ovary syndrome. Arterioscler Thromb Vasc Biol 1995:15(7):821-6.

8. Limper M, de Kruif MD, Duits AJ, Brandjes DP, van Gorp EC. The diagnostic role of procalcitonin and other biomarkers in discriminating infectious from noninfectious fever. J Infect 2010;60(6):409-16.

9. Linscheid P, Seboek D, Nylen E.S, Langer I, Schlatter M, Becker K.L, Keller U, Muller B. In vitro calcitonin I gene expression in parenchymal cells: a novel product of 
human adipose tissue. Endocrinology 2003;144(12): 5578-84.

10. Abbasi A, Corpelejin E, Postmus D, Gansevoort RT, de Jong PE, Gans RO, Struck J, Hillege HL, Stolk RP, Navis $\mathrm{G}$, Bakker SJL. Plasma procalcitonin is associated with obesity insulin resistance, and metabolic syndrome. J Clin Endocrinol Metab 2010;95(9):E26-E31.

11. Kershaw EE, Flier JS. Adipose tissue as an endocrine organ. J Clin Endocrinol Metab 2004;89(6):2548-56.

12. Linscheid P, Seboek D, Schaer DJ, Zulevski H, Keller U, Muller B. Expression and secretion of procalcitonin and calcitonin gene-related peptide by adherent monocytes and by macrophage-activated adipocytes. Crit Care Med 2004;32(8):1715-20.

13. Ferriman D, Gallwey JD. Clinical assessment of body hair growth in women. J Clin Endocrinol Metab 1961; $21: 1440-7$.

14. Friedewald WT, Levy RI, Fredrickson DS. Estimation of the concentration of low-density lipoprotein cholesterol in plasma, without use of the preparative ultracentrifuge. Clin Chem 1972;18(6):499-502.

15. Mak W, Dokras A. Polycystic ovarian syndrome and the risk of cardiovascular disease and thrombosis. Semin Thromb Hemost 2009; 5(7):613-620.

16. Mancini F, Cianciosi A, Reggiani GM, Facchinetti F, Battaglia C, de Aloysio D. Endothelial function and its relationship to leptin, homocysteine, and insulin resistance in lean and overweight eumenorrheic women and PCOS patients: A pilot study. Fertil Steril 2009;91(6):2537-44.

17. Sravan Kumar P, Ananthanarayanan PH, Rajendiran S. Cardiovascular risk markers and thyroid status in young Indian women with polycystic ovarian syndrome: A casecontrol study. J Obstet Gynaecol Res 2014;40(5):1361-7.

18. Salehpour S, Manzor-Al-Ajdad O, Samani EN, Abadi A. Evaluation of homocysteine levels in patients with polycystic ovarian syndrome. Int J Fertil Steril 2011; 4(4):16871 .

19. Lin YH, Huang SY, Hsu MI, Chang YC, Cheng CY, Hsu CS, Tzeng CR. Hyperhomocysteinaemia is associated with biochemical hyperandrogenaemia in women with reproductive age. Eur J Obstet Gynecol Reprod Biol 2013; 171(2): 314-8.

20. Maleedhu P, Vijayabhaskar M, Sharma SSB, Kodumuri PK, Vasundra Devi V. Status of homocysteine in polycystic ovary syndrome. J Clin Diagn Res 2014;8(2):31-3.

21. Kilic-Okman T, Guldiken S, Kucuk M. Relationship between homocysteine and insulin resistance in women with polycystic ovary syndrome. Endocr J. 2004;51(5):505-8.

22. Dinavahi R, Falkner B. Relationship of homocysteine with cardiovascular disease and blood pressure. J. Clin Hypertens 2004;6(9):494-8

23. Fulghesu A, Magnini R, Portoghese E, Angioni S,
Minerba L, Melis GB. Obesity-related lipid profile and altered insulin incretion in adolescents with polycystic ovary syndrome. J Adolesc Health 2010;46(5):474-81.

24. Sumithra NU, Lakshmi RL, LeelaMenon N, Subhakumari KN, Sheejamol VS. Evaluation of oxidative stress and hsCRP in polycystic ovarian syndrome in a tertiary care hospital. Indian J Clin Biochem 2015;30(2):161-6.

25. Pedroso DC, Miranda-Furtado CL, Kogure GS, Meola J, Okuka M, Silva C, Calado RT, Ferriani RA, Keefe DL, dos Reis RM. Inflammatory biomarkers and telomere length in women with polycystic ovary syndrome. Fertil Steril 2015;103(2):542-7.

26. Puder JJ, Varga S, Kraenzlin M, De Geyter C, Keller U, Müller B. Central fat excess in polycystic ovary syndrome: relation to low-grade inflammation and insulin resistance. J Clin Endocrinol Metab 2005;90(11):6014-21.

27. Steinwald PM, Whang KT, Becker KL, Snider RH, Jr, Nylen ES, White JC. Elevated calcitonin precursor levels are related to mortality in an animal model of sepsis. Crit Care 1999;3(1):11-16.

28. Becker KL, Snider R, Nylen. ES. Procalcitonin in sepsis and systemic inflammation: a harmful biomarker and a therapeutic target. Br J Pharmacol 2010;159(2):253-64.

29. Becker KL, Snider R and Nylen ES. Procalcitonin assay in systemic inflammation, infection and sepsis clinical utility and limitations. Crit Care Med 2008;36(3):941-952.

30. Repaci A, Gambineri A, Pasquali R. The role of low-grade inflammation in the polycystic ovary syndrome. Mol Cel Endocrinol 2011;335(1):30-41.

31. Rashad NM, El-Shal AS, Abdelaziz AM. Association between inflammatory biomarker serum procalcitonin and obesity in woman with polycystic ovary syndrome. J Reprod Immunol 2013;97(2):232-239.

32. Wyczalkowska-Tomasik A, Czarkowska-Paczek B, Zielenkiewicz M, Paczek L. Inflammatory markers change with age, but do not fall beyond reported normal ranges. Arch Immunol Ther Exp 2016;64(3):249-54.

33. Carbonnel C, Seux V, Pauly V, Oddoze C, Roubicek C, Larue JR, Thrion X, Soubeyrand J, Retornaz F. Estimation of the glomerular filtration rate in elderly inpatients comparison of four methods. Rev Med Interne 2008:29(5); 364-9.

34. Singh T, Newman AB. Inflammatory markers in population studies of aging. Ageing Res Rev 2011;10(3):319-29.

35. Capurso C, Solfrizzi V, D’Introno A, Colacicco AM, Capurso SA et al. Interleukin 6-174 G/C promoter gene and sporadic Alzheimer's disease: Geographic allele and genotype polymorphism variation in Europe. Exp Gerontol 2004;39(10):1567-73.

36. Szarc vel Szic K, Declerc K, Vidakovic M, Vanden Berghe W. From inflammaging to healthy aging by dietary lifestyle choices: Is epigenetics the key to personalized nutrition? Clin Epigenetics 2015; 25:7:33. 